\title{
The dimensions of Integrated Manufacturing Systems Engineering
}

\author{
P. Ladet ${ }^{1}, F$. Vernadat 2

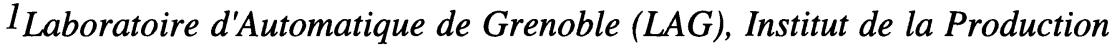 \\ Industrielle, ENSGI-INPG, 46 avenue Félix Viallet, F-38031 Grenoble, France \\ Phone: +33 765748 32; Fax: +33 765743 17; e-mail: joyaux@imag.fr \\ 2INRIA Rhône-Alpes, 46 avenue Félix Viallet, F-38031 Grenoble, France \\ Phone: +3376 5747 77; Fax: +3376 5747 54; \\ e-mail: Francois.Vernadat@inria.fr
}

\begin{abstract}
Modern, integrated manufacturing systems need to be engineered in a systematic way like any other complex dynamic systems. Due to the extreme complexity and interdisciplinarity nature of manufacturing system design, analysis, reengineering and continuous improvement, and due to the trend for internetworking of enterprises, a new discipline called Enterprise Engineering is emerging. Different aspects or dimensions of Enterprise Engineering in the context of integrated manufacturing systems engineering are reviewed in the paper.
\end{abstract}

\section{Keywords}

Enterprise Engineering, integrated manufacturing systems, interdisciplinarity aspects, integration, system life cycle, complexity

\section{INTRODUCTION}

Manufacturing enterprises play an essential role in industrialised countries both in terms of employment and revenues. Central to these are manufacturing systems, which have become significantly complex systems to design and to control. They can be defined as socio-economic discrete event dynamic systems.

Nowadays, the economic environment of most manufacturing enterprises is drastically changing. The economy of scale is being replaced by an economy of scope. Customisation, i.e. product adaptation to specific customer requirements, is driving the demand. Globalisation, i.e. the necessity to be present on world-wide markets, implies a timely deployment strategy in terms of product manufacturing and distribution, sometimes forcing strategic alliances with partner companies. Fierce competition with emerging countries forces industrialised countries to produce at lower cost, with higher quality and in shorter delays. Finally, the consequence of widespread automation and the need to gain on productivity are pushing manufacturing enterprises to lean their management and manufacturing operations, therefore employing less and less people. 
Proper design or reengineering of their manufacturing systems is now a must for most industrial companies to face international competition. Modern manufacturing systems must be:

- flexible/agile

- reactive

- integrated and

- cost-effective

Manufacturing systems, like any other type of complex systems, need to be designed and engineered in a systematic way by means of structured approaches relying on sound principles and supported by efficient tools and methods. We call such an emerging approach Enterprise Engineering.

This book only provides a first step in this direction and deals with:

- the concept of the Extended Enterprise

- some Enterprise Engineering approaches under development

- techniques for business process modelling and reengineering

- formal approaches for (integrated) manufacturing systems specification

- Petri net techniques for modelling and analysing the physical part of the manufacturing system as well as production planning aspects such as process planning

- techniques for manufacturing system coordination and integration (both at the plant level and at the enterprise level), and

- pre-normative and standardisation issues in the areas of enterprise modelling and integration

\section{ENTERPRISE ENGINEERING}

Enterprise Engineering is the art of designing, implementing, maintaining and continuously improving enterprise systems (processes and components) so that the enterprise can fulfil its mission according to its business objectives.

Enterprise Engineering embraces under one term several engineering disciplines such as industrial engineering, systems engineering, information systems engineering and production engineering. It also relies on techniques from management sciences, organisation sciences, applied mathematics (especially, simulation and Operations Research) or human resource management.

Traditionally, these various disciplines have been applied separately and in ad hoc ways when designing or reengineering some of the enterprise business processes or any part of the enterprise. The overall process remains essentially sequential (performed step by step, "throwing the project over the wall" from one engineering domain to the next one) and technical barriers, mainly due to protected "islands of competence", are typical attitudes encountered in manufacturing systems engineering departments. Organisation and human resource management aspects remain clearly separated from technical aspects such as functional design, information system design, manufacturing plant layout design or computer network system design. Today, most companies realise that management, organisational and technical issues are closely inter-related, especially in the context of integrated systems.

There is a need to break down these barriers and develop structured methodologies to be supported by models and computer tools to quickly and efficiently engineer/reengineer modern manufacturing systems. Such methodologies should help and guide system designers to:

- improve integration of enterprise components to increase enterprise productivity and efficiency on the basis of enhanced communication, cooperation and coordination of enterprise operations. This is the aim of CIM (Computer-Integrated Manufacturing);

- simplify and lean management and manufacturing procedures to gain on costs and delays; 
- parallelise work and operate according to the Just-In-Time (JIT) philosophy to reduce time-tomarket and reduce backlogs and work-in-process inventories; and

- capitalise on previous knowledge and know-how to learn from past experience and build the enterprise memory, as required by Continuous Process Improvement (CPI).

Methodologies supporting Enterprise Engineering must lead to solutions which help the enterprise to compete on quality, costs and delays (QCD) and to face management of change because of the fierce competition on markets in a rapidly changing world.

Several aspects or dimensions characterising Enterprise Engineering must be considered. In this paper, we focus on:

- the dimension of interdisciplinarity

- the dimension of system life cycle

- the dimension of integration

- the dimension of complexity

\section{THE DIMENSION OF INTERDISCIPLINARITY (OR VIEWS)}

The complexity of manufacturing systems, as systems created by humans for their own needs, has progressively increased to the point of being comparable to the complexity of natural systems. There are two reasons for this:

- their management and control functions are themselves becoming more and more complex as well as their integration needs; and

- the role of human operators in the control loop, recently rehabilitated after an era of over automation, brings a part of uncertainty into the control process. This is a dimension which is not easy to formalise.

The control of production systems in general, and of manufacturing systems in particular, relies on a representative modelling of these systems as well as the control and management policies to be applied to them. However, each function and each control level, due to its nature and the concepts handled, requires a priori a different modelling tool. For instance, a scheduling function, a machine control function, resource sharing and synchronisation, flow modelling on the shop floor or human operator integration, all call for different modelling and analysis tools.

An interdisciplinary approach for Enterprise Engineering can therefore be defined on a threelayer basis, corresponding to three levels of granularity in system modelling:

1. The first layer concerns the elementary level: A given function, a view of a manufacturing process, a system component are often themselves very complex. Their analysis assumes the availability of methods and modelling tools able to take into account their detailed specificities and intricacies to a level as close to reality as possible. This regularly leads to advanced enhancement of existing tools as well as to the definition of new tools, usually derived from previous ones. Advances in Petri nets provide a good example (see the Manufacturing System Analysis section of this book for more details).

2. The second layer concerns the level of tool cooperation: The tools used to represent a given function or a given mechanism must be able to exchange information, to be synchronised or to cooperate with one another as well as to take into account the function or mechanism environment, i.e. the other functional elements of the system. This is the problem of structured representation of an application perceived from different approaches and disciplinarity angles at different levels of abstraction, each one relying on a different perception of the system modelled in terms of a given tool. The problem is to be able to 
interface these tools by the definition of consistent information systems or even a unique information system to support collaborative decision making and engineering thinking.

3. The third layer concerns the global level: It consists in developing an all-embracing modelling environment able to represent the set of all facets of a manufacturing system or more precisely to federate tools which, although remaining different and specialised to the aspects they have been developed for, have been designed in a perspective for integrated analysis and design. This is an area still under development and it is a long way far from complete achievement. Relevant papers related to this area can be found in the Enterprise Engineering section of this book.

For each of these layers and throughout the system development life cycle, an interdisciplinary approach must be used for Enterprise Engineering in general, and for integrated manufacturing systems engineering in particular. This concerns engineering sciences on one hand and management sciences and human sciences on the other hand.

Too often, the design and reengineering of manufacturing systems is primarly considered as a matter of industrial engineering and application domain engineering (such as mechanical engineering, electrical engineering, or food industry engineering for instance), i.e. as a technical problem. In fact, it is also, and sometimes most of all, an organisational problem, a management problem, a human problem and an economic problem. In the case of integrated systems, it also becomes a problem for computer scientists and information system designers.

The challenge in Enterprise Engineering is to develop a framework supported by methodologies and tools to provide these different areas of expertise or viewswith

- a way to understand the "language" or viewpoint of other disciplines

- a way to indicate where and how each one fits in the framework

- a way to federate the viewpoints and expertises of each of them

so that they can operate in synergy to develop/reengineer enterprise systems faster, better and at a reasonable cost to meet specified business objectives.

\section{THE DIMENSION OF SYSTEM LIFE CYCLE}

The system life cycle covers the set of activities going from enterprise system inception to engineering, implementation, operation, improvement and finally system dismantlement.

In addition to the interdisciplinarity dimension, it is now widely understood that this process is not strictly sequential (it does not follow a "waterfall" approach) but involves a number of iterations, redoings and revisions at all levels. Furthermore, emerging principles of Concurrent Engineering (CE) principles can be applied to the design and engineering of enterprise systems (i.e. for an entire plant or just a subsystem of it). Moreover, since there is always something changing within the enterprise or in its environment, operational and management systems must be regularly analysed to be improved or corrected. These are the areas of strategic management on the administrative side and Continuous Process Improvement (CPI) on the technical side, both defining the needs for Business Process Reengineering (BPR).

New issues in enterprise systems engineering come from the pressure to pay more attention to environmental and energy issues in product and production system design and operation all over the system life cycle. Recycling of product components as well as production system dismantlement become important issues in systems engineering.

The major phases of enterprise system life cycle have been identified and documented by the Purdue Enterprise Reference Architecture (PERA) and the European pre-norm ENV 40003 Framework for Enterprise Modelling produced by Comité Européen de Normalisation (CEN). They involve: 
- Business Objectives and Mission Definition: This concerns strategic planning defined by top management and must answer such questions as: what will be produced, for which market segments, in what quantities and where.

- Requirements Definition: In this phase, business users must express in detail what has to be done to achieve business objectives.

- Design Specification: This phase covers preliminary design and detailed design of business processes, resource needs and management, information systems and infrastructure and organisation structures. Technology aspects as well as human factors must be considered in terms of required capabilities. Formal description and simulation techniques are extensively used. Exception handling mechanisms must be planned and analysed.

- Implementation Description: Decisions on resource selection and layout, data distribution, computer network configuration, etc. are made and documented in this phase.

- Installation: Components and programs of the system are implemented and tested.

- Operation: This phase is concerned with actual exploitation of the system in its business environment.

- Management of change/Continuous Process Improvement: This concerns the identification of shortcomings or defficiencies in the system and their correction.

- Dismantlement or shut down.

\section{THE DIMENSION OF INTEGRATION}

Integration means putting system components together to create a synergistic whole, the capabilities of which encompass the capabilities of each of its components alone. The benefits of integration rely on improved communications, cooperation and coordination (C3) of the business processes of the system.

In the case of integrated manufacturing systems, integration can be achieved at several levels:

- Computer systems integration: This is concerned with physical systems integration in terms of systems interconnections by means of computer networks. The 7-layer ISO-OSI architecture has been the technical reference but Ethernet is dominating the market of local area networks. In the near future, ATM is supposed to be the new standard both for local and wide area networks because of its high-speed and multimedia capabilities.

- Shop-floor integration: This is concerned with manufacturing systems integration and was originally initiated by the MAP (Manufacturing Automation Protocol) initiative in the US followed by the CNMA ESPRIT project in Europe, both based on the ISO-OSI architecture. The MMS (Manufacturing Message System) language is an important result of this work as well as fieldbus definitions (such as FIP and Profibus), which are simplified and faster computer network architectures dedicated for shop-floor applications.

- Plant integration: This form of integration goes one step beyond shop-floor integration and may involve the exchange of engineering data such as drawings and bills of materials. Thus, standard data exchange formats (such as IGES or STEP/EXPRESS for exchange of product and process data and EDI for administrative electronic data interchange) as well as common services are required to make applications communicate or even interoperate. Different kinds of integration platforms or integrating infrastructures known as middleware platforms are being proposed. Examples of generic integration platforms are OSF/DCE and OMG/CORBA. CCE-CNMA is an integration platform dedicated to CIM environments.

- Enterprise Integration: Enterprise Integration concerns the integration of the various business processes of the enterprise to facilitate information, material and decision flows and therefore increase reactivity and productivity. It deals with integration at the business level of the enterprise. It requires a model of the business processes (as provided by Enterprise 
Modelling) and an information infrastructure to monitor execution of this model and coordination of the business processes. These are the ideas promoted by CIMOSA, the European open system architecture for CIM.

- Extended Enterprise or inter-enterprise integration: Finally, the last level of integration concerns the Extended Enterprise. While CIM mostly concerns intra-enterprise integration (i.e. integration of the business processes of a given enterprise), the Extended Enterprise is concerned with inter-enterprise integration (i.e. internetworking of enterprises interacting along a common supply chain).

MMS and CIMOSA are covered in the Enterprise Engineering section of the book while the coordination, CCE-CNMA and integrating infrastructure issues are covered in the Manufacturing System Coordination and Integration section. The Extended Enterprise has a section of its own.

\section{THE DIMENSION OF COMPLEXITY}

Due to requirements for flexibility/agility, reactivity, integration and automation, manufacturing systems, and especially discrete manufacturing systems, become extremely complex systems to design and to control. Their complexity often largely exceeds the capabilities of one person. For instance, let us think about a manufacturing system producing gear-boxes for cars or trucks or an automated system producing electronic chips. Such systems are made of 10 to 20 workcenters, can produce over a hundred of different product types and may involve dozens of different concurrent business processes.

The complexity may come from the large number of system components and their interactions. It may also come from the sophistication of the system.

In terms of systems engineering, we have outlined the interdisciplinary aspects and the need for different types of models to correctly assess the systems. The mixing of competences (engineering, organisational, socio-economic, etc.) is also a factor of complexity in the design of such systems as well as in the design of relevant Enterprise Engineering methodologies.

Another aspect related to complexity concerns scalability of models. Currently, various techniques are proposed to model manufacturing systems, their business processes or their information systems. They are usually presented on small toy-examples in the literature. However, in industry people must deal with large models representing real systems. It is therefore important to consider the scalability aspects when proposing a new modelling approach.

\section{CONCLUSION}

Enterprise Engineering as defined in this paper is a collection of many different activities put together to engineer enterprise systems, and especially integrated manufacturing systems. The challenge of Enterprise Engineering is to provide industry with methods and guidelines for building efficient enterprise systems (processes and components) faster and better. There is therefore a need for developing advanced tools (models and computer tools) as well as suitable methodologies.

Development of these tools and methodologies must take into account the interdisciplinarity nature of such an engineering process, must cover the entire enterprise system life cycle, pay special attention to integration issues and provide mechanisms to face the complexity dimension of the problem.

The aim of this book is to provide a first step in this direction. Especially, it is shown how recent developments in Enterprise Modelling and Enterprise Integration can be combined with Business Process Reengineering (BPR) and manufacturing system specification and analysis 
techniques (such as formal description techniques and Petri nets) to achieve some of the goals of Enterprise Engineering.

More developments remain to be done to bring Enterprise Engineering to the level of maturity of Software Engineering for example, from which it borrows many ideas and adopts a similar approach. Especially, it is the authors' opinion that more work on formal techniques should be pursued to design robust and reliable systems so that their qualitative and quantitative properties can be more precisely analysed before they are installed and operated.

\section{BIOGRAPHY}

Pierre Ladet is a French citizen and lives in Grenoble. He got his Doctorat d'Etat degree in 1982 from the Institut National Polytechnique de Grenoble (INPG) in Physical sciences (Automatic control). He his currently a professor at INPG. He has been a research officer at the Automatic Control Laboratory of Grenoble (1982-1995) where he created a research team on discrete events systems.

His research interests include discrete events systems and decision rules modelling using Petri nets. With his research team, he developed a Petri nets methodology and automatic control architecture including scheduling, decision and control aspects.

Professor Pierre Ladet is currently head of the national programme of CNRS « Conception des systèmes de production », an interdisciplinary research action which puts together disciplines from Ingineering Sciences and Human Sciences.

He also a mission officer for the French Ministry of Research and Education.

François Vernadat is a French and Canadian citizen. He got his Ph.D. degree in 1981 from the University of Clermont, France in Electronics and Automatic Control. From 1981 till 1988, he has been a research officer at the Division of Electrical Engineering of the National Research Council, Ottawa, Canada. In 1988, he joined INRIA, a French research institute in computer science and automatic control.

His research interests include CIM database technology and information systems, enterprise modeling and integration, knowledge representation, formal description techniques, Petri nets and manufacturing plant layout design. He has been involved in several ESPRIT projects and was one of the chief architects of CIMOSA. He has published over 90 scientific papers and is co-author of three books. He is the European editor for the International Journal of CIM and serves the scientific committee of several journals.

Dr. Vernadat served several times as a technical expert for the CIM program of the Commission of the European Communities (EU DG III). He acts as a French expert for national, European and international standardisation bodies on Enterprise Modelling and Integration (AFNOR, CEN TC 310, ISO TC 184). He is a member of the IEEE Computer Society, ACM and SME. 